\title{
Comparison of Image Quality, Myocardial Perfusion, and Left Ventricular Function Between Standard Imaging and Single- Injection Ultra-Low-Dose Imaging Using a High-Efficiency SPECT Camera: The MILLISIEVERT Study
}

\author{
Andrew J. Einstein ${ }^{1,2}$, Ron Blankstein ${ }^{3}$, Howard Andrews ${ }^{4}$, Mathews Fish ${ }^{5}$, Richard Padgett ${ }^{5}$, Sean W. Hayes ${ }^{6,7}$, \\ John D. Friedman ${ }^{6,7}$, Mehreen Qureshi ${ }^{1}$, Harivony Rakotoarivelo ${ }^{1}$, Piotr Slomka ${ }^{6}$, Ryo Nakazato ${ }^{6,8}$, Sabahat Bokhari ${ }^{1}$, \\ Marcello Di Carli ${ }^{3}$, and Daniel S. Berman ${ }^{6,7}$ \\ ${ }^{I}$ Department of Medicine, Cardiology Division, Columbia University Medical Center and New York-Presbyterian Hospital, New York, \\ New York; ${ }^{2}$ Department of Radiology, Columbia University Medical Center and New York-Presbyterian Hospital, New York, \\ New York; ${ }^{3}$ Department of Medicine, Cardiology Division, Brigham and Women's Hospital, Boston, Massachusetts; ${ }^{4}$ Department of \\ Biostatistics, Columbia University, New York, New York; ${ }^{5}$ Oregon Heart and Vascular Institute, Springfield, Oregon; ${ }^{6}$ Departments of \\ Imaging and Medicine and Cedars-Sinai Heart Institute, Cedars-Sinai Medical Center, Los Angeles, California; ${ }^{7}$ David Geffen \\ School of Medicine, University of California Los Angeles, Los Angeles, California; and ${ }^{8}$ Cardiovascular Center, St. Luke's \\ International Hospital, Tokyo, Japan
}

SPECT myocardial perfusion imaging plays a central role in coronary artery disease diagnosis, but concerns exist regarding its radiation burden. Compared with standard Anger SPECT (ASPECT) cameras, new high-efficiency (HE) cameras with specialized collimators and solid-state cadmium-zinc-telluride detectors offer potential to maintain image quality $(\mathrm{IQ})$, while reducing administered activity and thus radiation dose to patients. No previous study has compared $I Q$, interpretation, total perfusion deficit (TPD), or ejection fraction (EF) in patients receiving both ultralow-dose (ULD) imaging on an HE SPECT camera and standard low-dose (SLD) A-SPECT imaging. Methods: We compared ULD HE SPECT with SLD A-SPECT imaging by dividing the rest dose in 101 patients at 3 sites scheduled to undergo clinical A-SPECT myocardial perfusion imaging using a same day rest-stress ${ }^{99 \mathrm{mT}} \mathrm{Tc}$ protocol. Patients underwent HE SPECT imaging after an initial approximately $130-\mathrm{MBq}(3.5 \mathrm{mCi})$ dose and SLD-A-SPECT imaging after the remainder of the planned dose. Images were scored visually by 2 masked readers for IQ and summed rest score. TPD and EF were assessed quantitatively. Results: Mean activity was $134 \mathrm{MBq}(3.62$ $\mathrm{mCi}$ ) for ULD HE SPECT (effective dose, $1.15 \mathrm{mSv}$ ) and $278 \mathrm{MBq}$ (7.50 mCi, $2.39 \mathrm{mSv}$ ) for SLD A-SPECT. Overall IQ was superior for ULD HE SPECT $(P<0.0001)$, with twice as many studies graded excellent quality. Extracardiac activity and overall perfusion assessment were similar. Between-method correlations were high for summed rest score $(r=0.87)$, TPD $(r=0.91)$, and EF $(r=0.88)$. Conclusion: ULD HE SPECT rest imaging correlates highly with SLD A-SPECT. It has improved image quality, comparable extracardiac activity, and achieves radiation dose reduction to $1 \mathrm{mSv}$ for a single injection.

Received Jan. 28, 2014; revision accepted May 19, 2014.

For correspondence or reprints contact: Andrew J. Einstein, Columbia University Medical Center, 622 West 168th St., PH 10-203A, New York, NY 10032.

E-mail: andrew.einstein@columbia.edu

Published online Jun. 30, 2014.

COPYRIGHT (c) 2014 by the Society of Nuclear Medicine and Molecular Imaging, Inc.
Key Words: SPECT; radiation dose reduction; high-efficiency camera

J Nucl Med 2014; 55:1430-1437

DOI: 10.2967/jnumed.114.138222

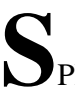
role in diagnosing patients with established or suspected coronary artery disease (CAD), in predicting outcomes, and in guiding management. However, concern has been raised regarding its radiation burden. In a landmark 2009 report, the National Council on Radiation Protection and Measurements noted a 6-fold increase in ionizing radiation exposure to the American population from medical procedures since the early 1980 s (1), in particular finding that more than $10 \%$ of the entire U.S. population radiation burden was related to MPI (2). The concern raised by this high radiation burden underscores the importance of efforts to keep radiation exposure as low as possible and, in particular, has generated considerable interest in developing methods to reduce radiation dose to patients from MPI, while preserving its benefits.

Based on a design advanced by Berkeley electrical engineer Hal Anger (3) in 1957, conventional Anger SPECT (A-SPECT) cameras now typically have 2 large thallium-doped sodium-iodide $(\mathrm{NaI}(\mathrm{Tl}))$ crystal detectors coupled to arrays of photomultiplier vacuum tubes. Used with low-energy, high-resolution collimators, these traditional cameras are able to detect only less than $0.02 \%$ of photon events (4). In contrast, new high-efficiency (HE) cameras incorporate multiple solid-state cadmium-zinc-telluride detectors arrayed surrounding the patient with a collimator geometry designed to optimize photon detection (5). Two such HE cameras have been introduced into clinical practice and offer potential to maintain image quality, while reducing administered activity and thus radiation dose to patients. HE SPECT imaging acquires up to 8 times as many photon counts per minute as does conventional A-SPECT (6), an advantage offering the potential to improve 
image quality, decrease image acquisition time, or decrease radiation dose. Several previous studies have evaluated the performance of MPI using an HE SPECT camera. These include studies of protocols with reduced administered activity and radiation dose $(7,8)$ and other studies comparing HE SPECT with A-SPECT imaging with equal doses of radiopharmaceutical (9-12). However, no previous study has validated reduced-dose MPI using an HE SPECT camera in comparison to traditional A-SPECT performed on the same patients. In this study, the MultIcenter nucLear Lowdose Imaging at a milliSIEVERT (MILLISIEVERT) Study, we directly compare image quality (IQ), interpretation, quantitative total perfusion deficit (TPD), and ejection fraction (EF) in patients who received both 1-mSv single-injection ultra-low-dose (ULD) imaging on one of the HE SPECT cameras and a standard protocol on an A-SPECT camera.

\section{MATERIALS AND METHODS}

\section{Study Design}

At 3 centers, in 101 patients with suspected or known CAD and scheduled to undergo rest-stress 1 -d ${ }^{99 \mathrm{~m} T c-b a s e d ~ M P I, ~ w e ~ d i v i d e d ~ t h e ~}$ rest dose so as to perform ULD (130 MBq [3.5 mCi]) rest imaging on an HE camera, followed by standard low-dose (SLD, 260-480 MBq [7-13 $\mathrm{mCi}$ ] of ${ }^{99 \mathrm{~m}} \mathrm{Tc}$ depending on standard clinical protocol) rest imaging on a conventional A-SPECT camera. Images were scored visually by 2 masked readers for IQ, extracardiac activity, and summed rest score (SRS), whereas TPD and EF were assessed quantitatively. These measures were statistically compared between ULD HE SPECT imaging and SLD A-SPECT imaging.

\section{Patient Population}

We prospectively enrolled patients at 3 sites (Cedars-Sinai Medical Center, Sacred Heart Medical Center, and Brigham and Women's Hospital) who were scheduled to undergo SPECT MPI for clinical indications. Patients were excluded if they had uncontrolled heart failure, uncontrolled hypertension, or obesity (body mass index $[\mathrm{BMI}]>30 \mathrm{~kg} / \mathrm{m}^{2}$ ). By prespecification, to ensure sufficient patients with resting perfusion

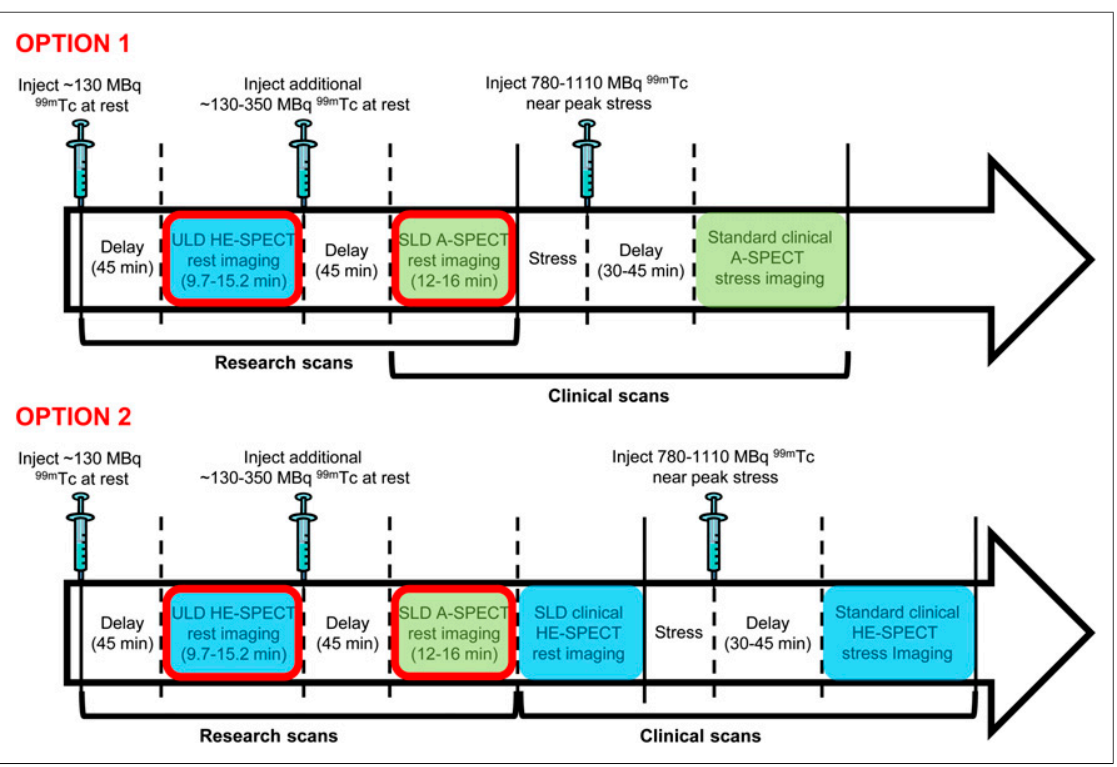

FIGURE 1. Study protocol. Sites had option to perform standard clinically indicated imaging using either A-SPECT camera (option 1) or HE SPECT camera (option 2). Images obtained using HE SPECT camera are denoted with blue shading and using A-SPECT camera with green shading. Comparison is made in each of the 2 options between images circled in red; subsequent images were obtained solely for clinical purposes and not analyzed in this study. defects, we planned to enroll 2 patient groups, each with 50 patients. Patient group 1 constituted patients without history of flow-limiting CAD (no known prior myocardial infarction or coronary revascularization), assessed by a treating physician as having intermediate or high pretest likelihood of CAD. Group 2 constituted patients with a history of myocardial infarction, specifically hospitalization for acute myocardial infarction or Q waves consistent with one. The study was registered (NCT01135095) and approved by the Institutional Review Boards of each institution; all subjects provided written informed consent.

\section{Imaging Protocol}

The imaging protocol is illustrated in Figure 1. For each patient, both ULD HE SPECT rest imaging and SLD A-SPECT rest imaging were obtained. Although site investigators had the option to choose ${ }^{99 \mathrm{~m}} \mathrm{Tc}$-sestamibi or ${ }^{99 \mathrm{~m}} \mathrm{Tc}$-tetrofosmin, all studies were performed using ${ }^{99 \mathrm{~m}} \mathrm{Tc}$-sestamibi. ULD rest imaging was performed with the patient supine, about $45 \mathrm{~min}$ after administration of approximately 130 $\mathrm{MBq}(3.5 \mathrm{mCi})$ of ${ }^{99 \mathrm{~m}} \mathrm{Tc}$-sestamibi, using a D-SPECT (Spectrum Dynamics) HE SPECT camera. Image acquisition time was determined using the patient's BMI, with a scheme designed by a medical physicist to ensure that a minimum of 700,000 left ventricular (LV) scintigraphic counts would be recorded (13). For patients with BMIs of $20-22 \mathrm{~m} / \mathrm{kg}^{2}$, acquisition time was $9.7 \mathrm{~min}$; for $22-24 \mathrm{~m} / \mathrm{kg}^{2}$, $10.7 \mathrm{~min}$; for $24-26 \mathrm{~m} / \mathrm{kg}^{2}, 13.0 \mathrm{~min}$; for $26-28 \mathrm{~m} / \mathrm{kg}^{2}, 14.1 \mathrm{~min}$; and for $28-30 \mathrm{~m} / \mathrm{kg}^{2}, 15.2 \mathrm{~min}$. Immediately after ULD HE SPECT rest imaging, an additional $130-350 \mathrm{MBq}(3.5-9.5 \mathrm{mCi})$ of ${ }^{99 \mathrm{~m} T c-}$ sestamibi were supplemented to achieve the planned rest dose of 260 $480 \mathrm{MBq}(7-13 \mathrm{mCi})$ prescribed for the patient by the local investigator, for the clinically indicated MPI study. After another delay of approximately $45 \mathrm{~min}$, SLD A-SPECT imaging was performed using the site's standard acquisition protocol, with images acquired over 1216 min. SLD A-SPECT rest imaging was performed at Cedars-Sinai using a Siemens ECAM or Philips Forte gamma camera, at Sacred Heart using a Philips Forte, and at Brigham and Women's using a Siemens Symbia T-6. All A-SPECT images were processed using the site's standard A-SPECT reconstruction protocol. In all 3 sites, this protocol uses iterative reconstruction; at Brigham and Women's Hospital, resolution recovery and scatter correction is performed as well (Flash 3D; Siemens).

The remainder of imaging was performed solely for clinical purposes, and these images were not evaluated as part of the research protocol. All patients underwent stress testing with injection of a clinically prescribed full dose (780-1,110 MBq [21-30 mCi]) of ${ }^{99 \mathrm{~m}} \mathrm{Tc}-$ sestamibi near peak stress and poststress ASPECT or HE SPECT imaging as clinically warranted.

\section{Administered Activity and Radiation Effective Dose Estimation}

Administered activity was measured using a dose calibrator immediately before administration of the ULD injection, and residual activity in the syringe/needle was measured immediately afterward. Received activity was determined as the difference between administered and residual activity. Similarly, administered, residual, and received activities were determined for the supplemental SLD injection. Total activities for the SLD injection were determined as the sum of ULD 
activities, decayed by the time between injections, and supplemental activities, using the equation

$$
\begin{aligned}
\text { Total SLD activity }= & \text { ULD activity } \\
& \times \mathrm{e}^{-0.693 \times \text { time between injections (hours) } / 4.8} \\
& + \text { supplemental SLD activity, }
\end{aligned}
$$

where $4.8 \mathrm{~h}$ is ${ }^{99 \mathrm{~m}} \mathrm{Tc}$ 's effective half-life.

Effective dose of radiation was estimated from received activities using a conversion factor based on the most recent organ dosimetry for 99mTc-sestamibi (14) and updated tissue-weighting factors (15).

\section{Assessment of Image Quality}

Deidentified images were transferred to the nuclear core laboratory at Cedars-Sinai. Each ULD HE SPECT and SLD A-SPECT set of images was assessed for image quality and semiquantitative image analysis by 2 board-certified nuclear cardiologists, each having read more than 50,000 cases, masked to the clinical and stress data. Readers were also masked to camera type, although in principle they could have predicted camera type for a study based on visual appearance of the perfusion imaging. HE SPECT and A-SPECT images for a given patient were read at separate times and in random order. A full dataset, including rotating projection image data, perfusion images, and gated images, was available to each reader for independent assessments. Each reader assessed overall IQ on a 5point scale (5, excellent; 4 , good; 3 , fair; 2 , poor; and 1 , uninterpretable) as well as the amount of extracardiac activity on a 5-point scale $(0$, none; 1, minimal/without any interference with scan interpretability; 2, mild/probably without any interference with interpretability; 3 , moderate/probably interfering with scan interpretability; and 4, severe/definitely interfering with scan interpretability). Where there was a discrepancy between readers, they subsequently reviewed the images jointly and assigned a consensus classification.

\section{Semiquantitative Image Analysis}

Each of the 2 nuclear cardiologists, as above, also visually assessed each ULD HE SPECT and SLD A-SPECT set of images for overall and segmental perfusion. Overall perfusion was assessed on a 5-point scale (5, normal; 4, probably normal; 3 , equivocal; 2 , probably abnormal; 1 , abnormal; and -, uninterpretable). Where there was a discrepancy between readers, the readers reviewed the images jointly and assigned a consensus classification. Segmental perfusion was assessed using a 17 -segment model (16) and a $0-4$ scale $(0$, normal uptake; 1 , mildly reduced uptake; 2 , moderately reduced uptake; 3 , severely reduced uptake; and 4, no uptake). Scoring was guided by boundaries overlaid on the SPECT slices by quantitative perfusion SPECT, and SRS was calculated by summing scores of the 17 segments. Additionally, to compare agreement between ULD HE SPECT and SLD A-SPECT interpretation with intrareader reproducibility of SLD A-SPECT interpretation, one of the readers read each study a second time, 3 mo after completion of the initial reads, and with cases presented in random order. Subsequently, the 2 readers reviewed images jointly and assigned a consensus classification to resolve any discrepancies.

\section{Quantitative Image Analysis}

TPD, a metric combining defect extent and severity as a percentage of total myocardium, was obtained by a core laboratory technologist, masked to clinical data, from ULD HE SPECT and SLD A-SPECT perfusion images using previously developed normal limits (17). The only manual step is adjustment of LV contours, if required, in a few cases. Separate camera-specific normal limits were applied for ULD HE SPECT and SLD A-SPECT images. EF was determined from gated short-axis images using an optimized version of Quantitative Gated SPECT (Cedars-Sinai), with 16 frames for A-SPECT and 8 for
HE SPECT. Additionally, for patients imaged at Cedars-Sinai, LV scintigraphic counts were determined from planar projections using a previously described method (13).

\section{Statistical Analysis}

Continuous and ordinal variables are described by median and interquartile range (IQR), or mean $\pm \mathrm{SD}$, and were compared using Wilcoxon signed-rank tests or correlated using the Pearson correlation coefficient. Agreement is displayed graphically using scatterplots and Bland-Altman plots. Intrareader, interreader, and between-method agreement of SRS were assessed by percentage agreement and weighted $\kappa$, with linear weighting. Statistical analysis was performed using Stata/SE (StataCorp).

\section{Study Approval and Role of Funding Source}

The study was conceived and initiated by the principal and senior investigators (AJE and DSB), who formulated the study design, with technical assistance (viz. acquisition time calculations) provided by a physicist employed by the funding source (Spectrum Dynamics). The decision as to the final study design remained solely that of the principal and senior investigators, with input from site investigators. At no point did the funding source have access to clinical or imaging data. The funding source played no role in the decision to publish or the content of the publication. The funding source remained masked to the results of the study until it was accepted for publication, except for its publicly available ACC.13 abstract.

\section{RESULTS}

\section{Patients and Doses}

The characteristics of the 101 patients enrolled are summarized in Table 1. Administered activities and radiation effective doses

\begin{tabular}{|c|c|}
\hline Characteristic & Value \\
\hline Total subjects & 101 \\
\hline \multicolumn{2}{|l|}{ Group } \\
\hline 1: intermediate/high likelihood of CAD & $55(54.5)$ \\
\hline 2: prior myocardial infarction & $46(45.5)$ \\
\hline \multicolumn{2}{|l|}{ Site } \\
\hline Cedars-Sinai & $49(48.5)$ \\
\hline Sacred Heart & $39(38.6)$ \\
\hline Brigham and Women's & $13(12.9)$ \\
\hline Mean age $\pm \mathrm{SD}(\mathrm{y})$ & $63.8 \pm 11.3$ \\
\hline Women & $47(46.5)$ \\
\hline BMI $\left(\mathrm{kg} / \mathrm{m}^{2}\right)$ & $26.1 \pm 2.8$ \\
\hline Range & $17.1-30.9$ \\
\hline Diabetes mellitus & $26(25.7)$ \\
\hline On insulin & $7(6.9)$ \\
\hline On oral medications & $17(16.8)$ \\
\hline Hypertension & $74(73.3)$ \\
\hline Hyperlipidemia & 77 (76.2) \\
\hline Current smoking & $13(12.9)$ \\
\hline Family history of premature heart disease & $31(30.7)$ \\
\hline No risk factors & $3(3.0)$ \\
\hline \multicolumn{2}{|l|}{ Stress type } \\
\hline Exercise: Bruce Protocol & $46(45.5)$ \\
\hline Exercise: Modified Bruce Protocol & $4(4.0)$ \\
\hline Adenosine & $25(24.8)$ \\
\hline Regadenoson & $25(24.8)$ \\
\hline Dobutamine & $1(1.0)$ \\
\hline
\end{tabular}

TABLE 1

Patient Characteristics

Data in parentheses are percentages. 
TABLE 2

Activities and Radiation Effective Doses

\begin{tabular}{|c|c|c|c|}
\hline Quantity & Mean & SD & Range \\
\hline \multicolumn{4}{|l|}{ ULD injection } \\
\hline Administered activity in $\mathrm{MBq}$ & $198(5.35)$ & $37(1.00)$ & $127-275(3.44-7.44)$ \\
\hline Residual activity in $\mathrm{MBq}$ & $64(1.74)$ & $26(0.71)$ & $6.3-144(0.17-3.89)$ \\
\hline Received activity in $\mathrm{MBq}$ & $134(3.62)$ & $28(0.75)$ & $67-189(1.80-5.10)$ \\
\hline Effective dose, received (mSv) & 1.15 & 0.24 & $0.57-1.63$ \\
\hline \multicolumn{4}{|c|}{ Supplemental injection, before SLD imaging } \\
\hline Administered Activity in MBq & $229(6.21)$ & $105(2.84)$ & $120-588(3.23-15.89)$ \\
\hline Residual activity in $\mathrm{MBq}$ & $65(1.75)$ & $46(1.24)$ & 5.9-270 (0.16-7.29) \\
\hline Received activity in $\mathrm{MBq}$ & $165(4.46)$ & $68(1.83)$ & $75-383(2.04-10.34)$ \\
\hline Effective dose, received (mSv) & 1.42 & 0.58 & $0.65-3.30$ \\
\hline \multicolumn{4}{|c|}{ Total SLD (supplemental injection + decayed ULD injection) } \\
\hline Received activity in MBq & $277(7.50)$ & $74(1.99)$ & $147-497(3.98-13.43)$ \\
\hline Effective dose, received (mSv) & 2.39 & 0.64 & $1.27-4.29$ \\
\hline
\end{tabular}

Data in parentheses are $\mathrm{mCi}$.

are summarized in Table 2 . The mean received activity from ULD injections was $134 \mathrm{MBq}(3.62 \mathrm{mCi})$, corresponding to an effective dose of $1.15 \mathrm{mSv}$.

\section{Count Statistics and Image Quality}

A mean of $1.34 \pm 0.51$ million LV counts were obtained for ULD HE SPECT images. There were more than 1 million counts for 36 of 49 patients (73\%), and an additional 7 patients (total $88 \%$ ) had more than 900,000 counts. On the basis of consensus reads, IQ was superior for ULD HE SPECT images in comparison to SLD A-SPECT images (Table 3), which was the case for patients in both patient groups. Extracardiac activity was similar between ULD HE SPECT and SLD A-SPECT, with borderline significantly less extracardiac activity noted in ULD HE SPECT images. Illustrative images comparing submillisievert ULD HE SPECT and SLD A-SPECT are shown in Figure 2.

\section{Perfusion Comparison}

Abnormal or probably abnormal rest perfusion imaging on SLD A-SPECT was noted in 22 patients, of which 18 were in group 2; equivocal rest perfusion in 11 patients; and normal or probably normal rest perfusion in 68 patients. Overall perfusion assessment, based on consensus of the 2 readers on the 5-point scale, was similar and not significantly different $(P=0.19)$ between ULD HE SPECT (median, 4; IQR, 3-5; mean \pm SD, $3.92 \pm 1.39$ ) and SLD A-SPECT (median, 4; IQR, 3-5; mean \pm SD, $3.76 \pm 1.50$ ).

SRS was not significantly different $(P=0.96)$ between ULD HE SPECT (mean $\pm \mathrm{SD}, 2.75 \pm 5.41)$ and SLD A-SPECT (mean \pm $\mathrm{SD}, 2.95 \pm 5.40)$ and was strongly correlated between the 2 methods ( $r=0.87, P<0.0001$ ), with few cases with notable differences in SRS (Fig. 3). The strong correlation $(r=0.84, P<0.0001)$ and lack of significant difference ( $P=0.63$ ) (ULD HE SPECT: median, 2 ; IQR, 1-6; mean \pm SD, $5.19 \pm 6.69$; and SLD-A-SPECT: median, 2; $\mathrm{IQR}, 2-8$; mean $\pm \mathrm{SD}, 5.58 \pm 6.51)$ remained when considering only abnormal studies, defined here as those 52 patients with an SRS greater than 1 using either method. There was outstanding interreader reproducibility in SRS, which was comparable to intrareader reproducibility. Both interreader and intrareader reproducibility were slightly better for ULD HE SPECT imaging than for SLD A-SPECT imaging (Table 4).

Median TPD was $1.8 \%$ (IQR, 0.5\%-4.6\%; mean, $4.5 \%$ ) for ULD HE SPECT and 2.6\% (IQR, 1.0\%-5.3\%; mean, $5.3 \%$ ) for
SLD A-SPECT, a difference that was statistically significant $(P=$ 0.04 ) but whose small magnitude is not clinically significant. There were few cases with notable differences in TPD between ULD HE SPECT and SLD A-SPECT (Fig. 3). For the 34 patients with a TPD greater than $5 \%$, this difference was not statistically significant (ULD HE SPECT: median, 6.1\%; IQR, 3.3\%-16.6\%; mean, $10.8 \%$; and SLD-A-SPECT: median, $10.1 \%$; IQR, 5.3\%-18.8\%; mean, $12.2 \% ; P=0.15)$. There was strong correlation in TPD between ULD HE SPECT and SLD A-SPECT (Fig. 3; $r=0.91$, $P<0.0001$ for all patients; $r=0.88, P<0.0001$ for patients with $\mathrm{TPD}>5 \%$ ).

\section{Function Comparison}

In one subject, no gated imaging was performed, and in another subject, there was a gating artifact that caused an erroneously calculated EF. For the remaining 99 cases, EF was statistically but not clinically significantly different between ULD HE SPECT (median, 62.9\%; IQR, 51.2\%-69.9\%; mean,

TABLE 3

Image Quality and Extracardiac Activity

\begin{tabular}{|c|c|c|}
\hline Quantity & ULD HE SPECT & SLD A-SPECT \\
\hline \multicolumn{3}{|l|}{$\mathrm{IQ}$} \\
\hline Excellent & 48 & 24 \\
\hline Good & 41 & 48 \\
\hline Fair & 5 & 22 \\
\hline Poor & 7 & 7 \\
\hline Mean score \pm SD & $4.29 \pm 0.85$ & $3.88 \pm 0.85$ \\
\hline$P$ (Wilcoxon) & \multicolumn{2}{|c|}{$<0.0001$} \\
\hline \multicolumn{3}{|l|}{ Extracardiac activity } \\
\hline None & 55 & 40 \\
\hline Minimal & 27 & 35 \\
\hline Mild & 10 & 19 \\
\hline Moderate & 3 & 2 \\
\hline Severe & 6 & 5 \\
\hline Mean score \pm SD & $0.79 \pm 1.12$ & $0.98 \pm 1.06$ \\
\hline$P$ (Wilcoxon) & \multicolumn{2}{|c|}{0.05} \\
\hline
\end{tabular}

Values are for consensus read. 


\begin{tabular}{|c|c|c|c|c|c|c|c|c|c|c|c|c|}
\hline & & \multicolumn{3}{|c|}{ Short axis } & \multicolumn{2}{|c|}{ Long axis } & \multicolumn{4}{|c|}{ Image assessment } & \multicolumn{2}{|c|}{ Received dose } \\
\hline & & Apical & Mid & Basal & Vertical & Horizontal & $\begin{array}{c}\text { Study } \\
\text { quality }\end{array}$ & Extracardiac activity & SRS & $\begin{array}{c}\text { Total perfusion } \\
\text { defect (\%) }\end{array}$ & $\begin{array}{c}\text { Activity } \\
\text { (MBq) }\end{array}$ & $\begin{array}{c}\text { Effective } \\
\text { dose } \\
\text { (mSv) }\end{array}$ \\
\hline $\begin{array}{l}\text { Brigham } \\
\text { and } \\
\text { ands }\end{array}$ & 罢 & & & & & & Excellent & None & 18 & 27.2 & 134 & 1.15 \\
\hline case 003 & 㟧 & & & & & & Good & $\begin{array}{l}\text { Mild and probably without } \\
\text { any interference with scan } \\
\text { interpretability }\end{array}$ & 14 & 23.2 & 497 & 4.29 \\
\hline $\begin{array}{l}\text { Cedars- } \\
\text { Sinai }\end{array}$ & 峁 & & & & & & Excellent & None & 5 & 6.3 & 134 & 1.16 \\
\hline case 033 & $\frac{\omega}{5}$ & & & & & & Good & None & 4 & 4.4 & 248 & 2.14 \\
\hline
\end{tabular}

FIGURE 2. Comparison of representative images between ULD HE SPECT and SLD A-SPECT imaging.

60.5\%) and SLD A-SPECT (median, 63.6\%; IQR, 57.9\%$73.1 \%$; mean, 64.0\%), with strong correlation (Fig. 3C; $r=$ $0.88, P<0.0001)$.

\section{Agreement Between ULD HE SPECT and SLD A-SPECT}

Agreement in SRS for SLD A-SPECT between 2 remote reads of a single reader was outstanding, with $95.7 \%$ agreement and a weighted $\kappa$ of 0.79 . Agreement in SRS between the reader's first SLD A-SPECT reading and ULD HE SPECT reading showed nearly as good agreement, with $94.4 \%$ agreement and a weighted $\kappa$ of 0.69 (Fig. 4). When analysis was limited to abnormal studies with an SRS greater than 1, findings were similar, with agreements $92.9 \%$ versus $87.8 \%$ and weighted $\kappa$ of 0.74 versus 0.58 , respectively (Table 4 ).

\section{DISCUSSION}

The introduction of high-sensitivity cameras has opened the possibility of reducing radiation dose associated with SPECT MPI. Although previous studies have evaluated HE SPECT protocols with reduced administered activity $(7,8)$, or compared HE SPECT with A-SPECT imaging using the same injected activities of radiopharmaceuticals (9-12), no previous study evaluated reduced-dose MPI using an HE SPECT camera in comparison to traditional A-SPECT performed on the same patients. Our multicenter study found that a single-injection SPECT MPI study can be performed using an HE SPECT camera at a mean radiation dose of $1 \mathrm{mSv}$, while attaining high correlation with conventional A-SPECT in terms of perfusion and function, with improved image quality and comparable extracardiac activity.

These results suggest that a stress-only procedure can be performed using an HE SPECT camera with effective dose of 1 $\mathrm{mSv}$. Single-injection rest imaging was chosen as the method with which to explore the low-radiation-dose procedure because it made it possible to perform ULD HE SPECT imaging under conditions identical to SLD A-SPECT imaging, using a divided rest dose. A stress dose cannot be analogously administered in a divided fashion but rather would require patients to undergo stress testing twice; differences between the 2 stress tests, for example, in patient hemodynamics or duration of exercise, might well result in differences in stress perfusion imaging that do not reflect any innate difference between a ULD injection imaged on an HE SPECT camera and a standard injection imaged on an ASPECT camera. The pharmacokinetics of ${ }^{99 \mathrm{~m}} \mathrm{Tc}$-sestamibi are similar at rest and stress, in fact with the proportion of the injected dose that is retained in the myocardium
FIGURE 3. Comparison of SRS from consensus reading, TPD, and resting EF between SLD ASPECT and ULD HE SPECT imaging. (A) SRS. (B) TPD (\%). (C) EF (\%). Top are scatterplots, and bottom are Bland-Altman plots. SEE denotes standard error of the estimate. 
TABLE 4

Agreement in Summed Rest Scores (SRS)*

\begin{tabular}{|c|c|c|c|c|}
\hline \multirow{2}{*}{ Reads compared } & \multicolumn{2}{|c|}{ All studies } & \multicolumn{2}{|c|}{ Abnormal studies $(\mathrm{SRS}>1)$} \\
\hline & SLD A-SPECT & ULD HE SPECT & SLD A-SPECT & ULD HE SPECT \\
\hline \multicolumn{5}{|l|}{ Intrareader agreement } \\
\hline Reader 1 , two reads & $0.79(95.7)$ & $0.83(96.7)$ & $0.74(92.9)$ & $0.78(93.7)$ \\
\hline \multicolumn{5}{|l|}{ Interreader agreement } \\
\hline Reader 1 first read vs. Reader 2 & $0.82(96.5)$ & $0.84(97.1)$ & $0.74(92.2)$ & $0.79(93.4)$ \\
\hline Reader 1 second read vs. Reader 2 & $0.78(95.5)$ & $0.85(96.8)$ & $0.73(92.5)$ & $0.80(93.7)$ \\
\hline \multicolumn{5}{|l|}{ Between-method agreement } \\
\hline $\begin{array}{l}\text { Reader } 1 \text { first read SLD A-SPECT vs. } \\
\text { reader } 1 \text { first read ULD HE SPECT }\end{array}$ & \multicolumn{2}{|c|}{$0.69(94.4)$} & \multicolumn{2}{|c|}{$0.58(87.8)$} \\
\hline $\begin{array}{l}\text { Reader } 1 \text { second read SLD A-SPECT vs. } \\
\text { reader } 1 \text { second read ULD HE SPECT }\end{array}$ & \multicolumn{2}{|c|}{$0.63(92.0)$} & \multicolumn{2}{|c|}{$0.54(87.8)$} \\
\hline $\begin{array}{l}\text { Reader } 1 \text { first read SLD A-SPECT vs. } \\
\text { reader } 1 \text { second read ULD HE SPECT }\end{array}$ & \multicolumn{2}{|c|}{$0.65(93.2)$} & \multicolumn{2}{|c|}{$0.57(87.4)$} \\
\hline $\begin{array}{l}\text { Reader } 1 \text { second read SLD A-SPECT vs. } \\
\text { reader } 1 \text { first read ULD HE SPECT }\end{array}$ & \multicolumn{2}{|c|}{$0.62(92.4)$} & \multicolumn{2}{|c|}{$0.54(87.6)$} \\
\hline $\begin{array}{l}\text { Reader } 2 \text { SLD A-SPECT vs. } \\
\text { reader } 2 \text { ULD HE SPECT }\end{array}$ & \multicolumn{2}{|c|}{$0.61(92.8)$} & \multicolumn{2}{|c|}{$0.46(83.3)$} \\
\hline $\begin{array}{l}\text { Consensus read SLD A-SPECT vs. } \\
\text { consensus read ULD HE SPECT }\end{array}$ & \multicolumn{2}{|c|}{$0.62(93.0)$} & \multicolumn{2}{|c|}{$0.52(87.4)$} \\
\hline
\end{tabular}

slightly higher at stress $(14,18)$. Thus, we expect that agreement between ULD HE SPECT and SLD A-SPECT imaging should be similar for rest imaging and stress imaging. Furthermore, extracardiac activity interfering with image interpretation is higher with rest than with stress studies (19), suggesting that ULD stress studies performed with the HE SPECT camera might be of higher quality than we observed on rest studies.

Stress-only imaging has lower radiation dose and faster laboratory throughput than traditional protocols incorporating both rest and stress imaging. It has become especially important in light of recent publications finding that an increasing percentage ( $>90 \%$ in 1 report) of rest-stress studies are normal (20) and that outcomes of normal rest-stress and stress-only studies are identical $(21,22)$. Reflecting these observations, a stress-only procedure is preferred for many patients in current guidelines $(23,24)$. Thus, we believe our findings suggesting the feasibility of 1-mSv stressonly MPI will be of interest to a broad community of physicians performing and ordering nuclear imaging studies.

The radiation dose reduction of more than $50 \%$ observed here for ULD HE SPECT is in reference to a baseline where SLD ASPECT imaging was performed with an average received activity of $277 \mathrm{MBq}(7.50 \mathrm{mCi})$. This activity, which is lower than the lower end of the recommended range of activity for low-dose rest imaging in current guidelines (25), reflects practice in 3 expert laboratories, all of which use iterative reconstruction and one of which uses software reconstruction incorporating resolution recovery and noise reduction. Such reconstruction techniques, which are also incorporated in the HE SPECT camera, can improve image quality and diagnostic ability and contribute to reducing radiation dose to patients. The low doses observed here in both SLD A-SPECT and ULD HE SPECT imaging underscore the message that both software and hardware advances can be used to lower doses from SPECT MPI. In particular, laboratories without access to HE SPECT cameras can still consider use of advanced reconstruction software to lower doses to their patients (5).

For patients requiring both stress and rest imaging on the same day, guidelines suggest that the second dose of radiopharmaceutical should generally be 3-4 times the activity of the first dose (25), to minimize the problem of shine-through, wherein residual activity from the initial dose interferes with the interpretation of the second dose. Thus, the findings of our study suggest that a complete rest-stress or stress-rest HE SPECT study could be completed with an effective dose of less than $5 \mathrm{mSv}(1+3$ or 4 $\mathrm{mSv}$ ). Even further, initial data from Nkoulou et al. (26) suggest that when an HE SPECT camera is used, accurate assessment of ischemic myocardial disease can be performed with the second dose of radiopharmaceutical having the same activity as the first dose. This suggests the possibility of $1-\mathrm{mSv}$ stress-first imaging performed with an HE SPECT camera, followed only if abnormal by same-day $1-\mathrm{mSv}$ rest imaging. This approach would reduce the radiation burden from a complete, $1-\mathrm{d}$ stress and rest MPI study to less than a year's background radiation. Thus, the findings here may be foundational for future efforts to lower radiation dose to patients using a variety of protocols on the new generation of $\mathrm{HE}$ SPECT cameras.

An important issue raised by our study is the challenge posed in low-dose MPI in accurately delivering a desired activity (mCi). In contrast to ${ }^{201} \mathrm{Tl},{ }^{99 \mathrm{~m}} \mathrm{Tc}$-based radiopharmaceuticals are sticky, adhering to their syringes. Residual activities of ${ }^{99 \mathrm{~m} T c-s e s t a m i b i}$ in the syringe averaged around $70 \mathrm{MBq}(2 \mathrm{mCi})$ but ranged from less than $7 \mathrm{MBq}(0.2 \mathrm{mCi})$ up to nearly $150 \mathrm{MBq}(4 \mathrm{mCi})$ for a ULD injection. Although for high-dose injections, typically around $1,110 \mathrm{MBq}$ (30 $\mathrm{mCi}$ ), and even for SLD injections, typically $300-440 \mathrm{MBq}(8-12 \mathrm{mCi})(25)$, this variability in residual activity represents a modest fraction of the actual received activity, 

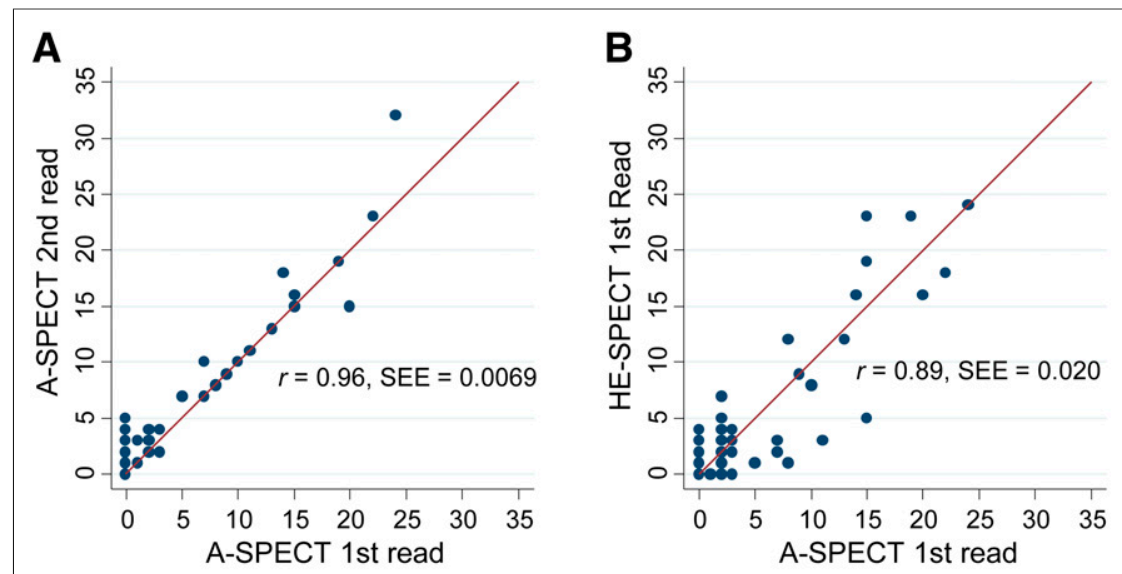

to examination of only 1 of the $2 \mathrm{HE}$ SPECT cameras currently available. One recent comparative study suggests that although both HE SPECT cameras have better performance characteristics than do Anger cameras, they differ in that the D-SPECT camera studied here has greater count sensitivity, whereas the other camera (Discovery NM 530c; GE Healthcare) has better spatial resolution and modestly higher contrast-to-noise ratio (4). A final limitation is that our images were limited to rest MPI. As discussed above, because we wanted to compare ULD HE SPECT with SLD A-SPECT MPI, evaluation of stress images would have required all patients to undergo stress testing on 2 separate occasions, because stress imaging cannot be performed with a divided dose as can rest imaging. We wanted both ULD HE SPECT and SLD ASPECT to be performed under identical conditions and thus chose to study the rest examination. However, as discussed above, the increased cardiac uptake and decreased extracardiac activity observed in stress images as compared with rest images suggest that our positive findings from ULD rest imaging are likely to be applicable to stress-only imaging as well.

FIGURE 4. Comparison of intrareader agreement in SRS of SLD-A-SPECT to agreement between SLD A-SPECT and ULD HE SPECT imaging. (A) Intrareader agreement in SRS comparing 2 SLD A-SPECT reads, spaced 3 mo apart, for first reader. (B) Between-method agreement in SRS comparing SLD A-SPECT with ULD HE SPECT, for first reader. Top are scatterplots, and bottom are Bland-Altman plots.

\section{CONCLUSION}

Our study demonstrates that in nonobese individuals with a high-efficiency SPECT camera, MPI can be performed

for ULD imaging this residual dose can represent most of the administered activity. A better understanding of determinants of ${ }^{99 \mathrm{~m}} \mathrm{Tc}$ residual activity, and development of methods to ensure reproducibility of received activity, will be important to ensure the quality of ULD MPI.

Our study is not without limitations. We excluded obese patients with a BMI of greater than $30 \mathrm{~kg} / \mathrm{m}^{2}$, for whom data about the suitability of MPI with an HE SPECT camera is mixed. For example, Fiechter et al. (27) found nondiagnostic IQ in most patients with a BMI of 40 or greater due to image truncation and IQ less than good in a third of patients with a BMI of 35-39.5 undergoing multipinhole HE SPECT imaging. Our calculations performed before the study to determine acquisition times suggested that for a 1-mSv injection, an acquisition of more than $15 \mathrm{~min}$ would be required for obese patients to ensure adequate count statistics. Because $15 \mathrm{~min}$ is often used as a maximum acquisition time, beyond which many patients are unable to lie still and thus the frequency of motion artifacts increases, obese patients were not included in this study aiming for a radiation dose of $1 \mathrm{mSv}$. Thus, the generalizability of our ULD HE SPECT protocol to obese patients remains to be demonstrated. Moreover, few patients were determined to have an EF less than $30 \%$, and thus further validation is required in this population. Another limitation is that the study was confined at high quality with an effective dose of $1 \mathrm{mSv}$ for a single injection.

\section{DISCLOSURE}

The costs of publication of this article were defrayed in part by the payment of page charges. Therefore, and solely to indicate this fact, this article is hereby marked "advertisement" in accordance with 18 USC section 1734. This study was supported by a grant from Spectrum Dynamics. Dr. Einstein has received grants for other research from GE Healthcare and Philips Healthcare. Drs. Berman and Friedman are shareholders in Spectrum Dynamics. Dr. Fish serves as a consultant for Spectrum Dynamics. No other potential conflict of interest relevant to this article was reported.

\section{ACKNOWLEDGMENTS}

We thank Dr. Lynne Johnson for helpful comments on the manuscript, Ming Feng of the Columbia University Data Coordinating Center for developing and managing the web-based system used to collect and manage clinical data, Jim Gerlach of the imaging core at Cedars-Sinai for assistance with image management and processing, and Nathaniel Roth of Spectrum Dynamics for medical physics calculations to determine the 
prescribed acquisition times. Dr. Einstein was supported in part as a Victoria and Esther Aboodi Cardiology Researcher and by a Herbert Irving Associate Professorship, the Louis V. Gerstner, Jr. Scholars Program, and the National Center for Advancing Translational Sciences, National Institutes of Health, through grant KL2 TR000081, formerly the National Center for Research Resources, grant KL2 RR024157. Clinical data coordinating services were supported by the National Center for Advancing Translational Sciences, National Institutes of Health, through grant UL1 TR000040. The content is solely the responsibility of the authors and does not necessarily represent the official views of the NIH.

\section{REFERENCES}

1. National Council on Radiation Protection and Measurements. Report No. 160, Ionizing Radiation Exposure of the Population of the United States. Bethesda, MD: National Council on Radiation Protection and Measurements; 2009.

2. Einstein AJ. Effects of radiation exposure from cardiac imaging how good are the data? J Am Coll Cardiol. 2012;59:553-565.

3. Anger HO. Scintillation camera. Rev Sci Instrum. 1958;29:27-33.

4. Imbert L, Poussier S, Franken PR, et al. Compared performance of highsensitivity cameras dedicated to myocardial perfusion SPECT: a comprehensive analysis of phantom and human images. J Nucl Med. 2012;53:1897-1903.

5. Slomka PJ, Dey D, Duvall WL, Henzlova MJ, Berman DS, Germano G. Advances in nuclear cardiac instrumentation with a view towards reduced radiation exposure. Curr Cardiol Rep. 2012;14:208-216.

6. Sharir T, Ben-Haim S, Merzon K, Prochorov V, Dickman D, Berman DS. Highspeed myocardial perfusion imaging initial clinical comparison with conventional dual detector anger camera imaging. JACC Cardiovasc Imaging. 2008; 1:156-163.

7. Oddstig J, Hedeer F, Jogi J, Carlsson M, Hindorf C, Engblom H. Reduced administered activity, reduced acquisition time, and preserved image quality for the new CZT camera. J Nucl Cardiol. 2013;20:38-44.

8. Berman DS, Kang X, Tamarappoo B, et al. Stress thallium-201/rest technetium-99m sequential dual isotope high-speed myocardial perfusion imaging. JACC Cardiovasc Imaging. 2009;2:273-282.

9. Esteves FP, Raggi P, Folks RD, et al. Novel solid-state-detector dedicated cardiac camera for fast myocardial perfusion imaging: multicenter comparison with standard dual detector cameras. J Nucl Cardiol. 2009;16:927-934.

10. Sharir T, Slomka PJ, Hayes SW, et al. Multicenter trial of high-speed versus conventional single-photon emission computed tomography imaging: quantitative results of myocardial perfusion and left ventricular function. J Am Coll Cardiol. 2010;55:1965-1974.

11. Songy B, Lussato D, Guernou M, Queneau M, Geronazzo R. Comparison of myocardial perfusion imaging using thallium-201 between a new cadmium-zinctelluride cardiac camera and a conventional SPECT camera. Clin Nucl Med. 2011;36:776-780
12. Duvall WL, Croft LB, Ginsberg ES, et al. Reduced isotope dose and imaging time with a high-efficiency CZT SPECT camera. J Nucl Cardiol. 2011;18:847857.

13. Nakazato R, Berman DS, Hayes SW, et al. Myocardial perfusion imaging with a solid-state camera: simulation of a very low dose imaging protocol. $\mathrm{J} \mathrm{Nucl}$ Med. 2013;54:373-379.

14. International Commission on Radiological Protection. Radiation dose to patients from radiopharmaceuticals (addendum 2 to ICRP publication 53). ICRP publication 80. Ann ICRP. 1998;28:1-126.

15. International Commission on Radiological Protection. The 2007 recommendations of the International Commission on Radiological Protection. ICRP publication 103. Ann ICRP. 2007;37:1-332.

16. Cerqueira MD, Weissman NJ, Dilsizian V, et al. Standardized myocardial segmentation and nomenclature for tomographic imaging of the heart: a statement for healthcare professionals from the Cardiac Imaging Committee of the Council on Clinical Cardiology of the American Heart Association. Circulation. 2002; 105:539-542.

17. Slomka PJ, Nishina H, Berman DS, et al. Automated quantification of myocardial perfusion SPECT using simplified normal limits. J Nucl Cardiol. 2005; 12:66-77.

18. Wackers FJ, Berman DS, Maddahi J, et al. Technetium-99m hexakis 2-methoxyisobutyl isonitrile: human biodistribution, dosimetry, safety, and preliminary comparison to thallium-201 for myocardial perfusion imaging. $J$ Nucl Med. 1989;30:301-311.

19. Rehm PK, Atkins FB, Ziessman HA, et al. Frequency of extra-cardiac activity and its effect on 99Tcm-MIBI cardiac SPET interpretation. Nucl Med Commun. 1996;17:851-856.

20. Rozanski A, Gransar H, Hayes SW, et al. Temporal trends in the frequency of inducible myocardial ischemia during cardiac stress testing: 1991 to 2009. J Am Coll Cardiol. 2013;61:1054-1065.

21. Chang SM, Nabi F, Xu J, Raza U, Mahmarian JJ. Normal stress-only versus standard stress/rest myocardial perfusion imaging: similar patient mortality with reduced radiation exposure. J Am Coll Cardiol. 2010;55:221-230.

22. Duvall WL, Wijetunga MN, Klein TM, et al. The prognosis of a normal stressonly Tc-99m myocardial perfusion imaging study. J Nucl Cardiol. 2010;17: 370-377.

23. Depuey EG, Mahmarian JJ, Miller TD, et al. Patient-centered imaging. J Nucl Cardiol. 2012;19:185-215.

24. Cousins C, Miller DL, Bernardi G, et al. Radiological protection in cardiology. ICRP publication 120. Ann ICRP. 2013;42:1-125.

25. Henzlova MJ, Cerqueira MD, Mahmarian JJ, Yao SS. Stress protocols and tracers. J Nucl Cardiol. 2006;13:e80-e90. Updated at: http://www.asnc.org/imageuploads/ImagingGuidelinesStressProtocols021109.pdf.

26. Nkoulou R, Pazhenkottil AP, Kuest SM, et al. Semiconductor detectors allow low-dose-low-dose 1-day SPECT myocardial perfusion imaging. J Nucl Med. 2011;52:1204-1209.

27. Fiechter M, Gebhard C, Fuchs TA, et al. Cadmium-zinc-telluride myocardial perfusion imaging in obese patients. J Nucl Med. 2012;53:1401-1406. 